\title{
Control of neural synchrony with light activated opsins
}

\author{
Sachin S Talathi ${ }^{*}$, Shivakeshavan Ratnadurai ${ }^{2}$, Svetlana Kantorovich ${ }^{4}$, Paul R Carney ${ }^{1,3}$, Pramod P Khargonekar $^{2}$ \\ From Nineteenth Annual Computational Neuroscience Meeting: CNS*2010 \\ San Antonio, TX, USA. 24-30 July 2010
}

Synchronous neural activity occurs throughout the normal brain and has been associated with many important brain functions. Abnormal patterns of neural synchrony have been implicated in several neurological and psychiatric disorders such as epilepsy, autism, schizophrenia, and Parkinson's disease. Several attempts have been made over the last decade to suppress pathological neural synchrony through the use of pharmacological agents or electrical stimulation. However, these therapeutic techniques present significant technical challenges. For example, problems with pharmacological interventions include slow onset and offset, low target
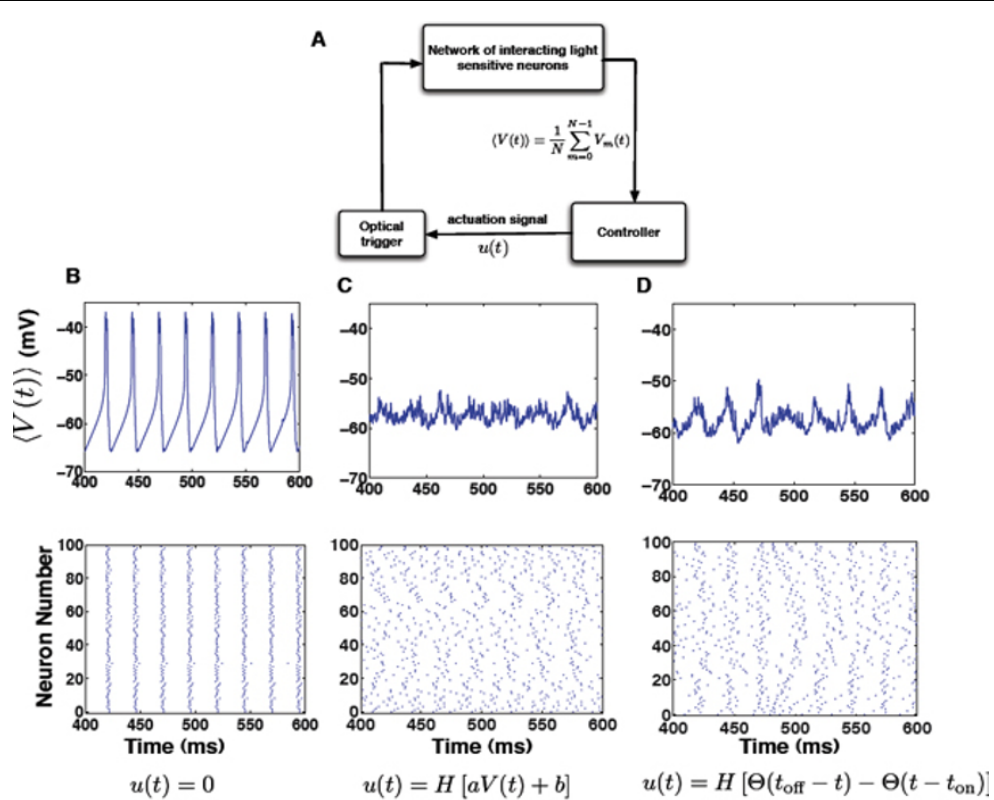

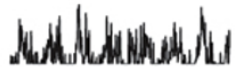

Figure 1 A) Schematic of the control architecture. The controller generates the feedback signal $u(t)$ that triggers an optical signal, which is feedback onto the genetically modified network. The light trigger activates Channelrhodopsin (ChR2) protein embedded into the genetically modified cell membrane resulting in depolarization of the neuron. B) The mean field network activity (top trace) and the raster activity (bottom trace) in absence of optical trigger. C) Network activity when $u(t)$ modulates the light intensity of optical stimulation. D) Network activity when $u$ ( $t$ ) triggers a light pulse. $\mathrm{H}$ : intensity of light; $\exists t_{o n} ;\left\langle V\left(t_{o n}-\tau\right)\right\rangle>V_{0}$ and $t_{\text {off }}=t_{o n}+W$, where $W$ is the width of the light pulse. Parameters $V 0, \tau, H$ and $W$ are determined by the controller. The parameters are: $V 0=-48 \mathrm{mV}, \tau=8 \mathrm{~ms}, H=0.9 \mathrm{~mW} / \mathrm{mm}^{2}, W=2 \mathrm{~ms}, a=0.125 \mathrm{mV}^{-1}$ and $b=7.25$.

\footnotetext{
* Correspondence: sachin.talathi@bme.ufl.edu

'Department of Biomedical Engineering, University of Florida, Gainesville, Florida, 32611, USA
} 
specificity and peripheral side effects. Problems with electrical stimulation include the lack of mechanistic understanding of the effect of the electrical stimulation on brain dynamics, trial and error methodology to determine effective stimulation parameters, limited battery life resulting in repeated invasive surgeries, and difficulty in producing purely inhibitory stimulus. Recent developments in the field of molecular and genetic biology now offer the opportunity to use optical techniques to modulate neuronal activity in vivo by making neurons light sensitive through the use of light activated opsins. Optical stimulation has potentially very significant advantages over previous techniques both in terms of temporal and spatial resolution. In addition, it allows for cell specific selective targeting to modulate neuronal activity.

Here, we investigate, in the setting of a computational simulation, a control strategy to modulate neural synchrony in a network of genetically engineered neurons that are sensitive to light stimulation. Our principle aim is to use the mean field signal $\langle V(t)\rangle$ generated by the network in the regime of stable network synchrony to generate an appropriate optical stimulation feedback signal $u(t)$ to suppress the network synchrony. The feedback signal is designed so as to achieve neural desynchrony with minimum variance between the intrinsic frequency of the individual neuron in a given network and the resulting frequency of the neuron oscillator in the desynchronized state of the network. The schematic diagram of the control architecture explored in this study is demonstrated in Figure 1A. Figure 1B represents the stable synchronous state of the genetically modified parvalbumin positive inhibitory neuronal network (all to all coupled) in absence of any feedback control. Figures 1B-1C provides examples of successful control of neural synchrony using two distinctly different forms of control signals.

\section{Acknowledgements}

This work was funded in part by the NIH grants R01-EB004752 and R01EB007082, the Wilder Center of Excellence for Epilepsy Research and Eckis Professor Endowment to PPK.

\section{Author details}

'Department of Biomedical Engineering, University of Florida, Gainesville, Florida, 32611, USA. ${ }^{2}$ Department of Electrical and Computer Engineering University of Florida, Gainesville, 32611, USA. ${ }^{3}$ Department of Pediatrics, University of Florida, Gainesville, 32611, USA. ${ }^{4}$ Department of Neuroscience, University of Florida, Gainesville, 32611, USA.

Published: 20 July 2010

doi:10.1186/1471-2202-11-S1-P5

Cite this article as: Talathi et al:: Control of neural synchrony with light activated opsins. BMC Neuroscience 2010 11(Suppl 1):P5.

\section{Submit your next manuscript to BioMed Central} and take full advantage of:

- Convenient online submission

- Thorough peer review

- No space constraints or color figure charges

- Immediate publication on acceptance

- Inclusion in PubMed, CAS, Scopus and Google Scholar

- Research which is freely available for redistribution 